\title{
Quantification of Blood Flow from Rotational Angiography
}

\author{
I. Waechter ${ }^{1}$, J. Bredno ${ }^{2}$, D.C. Barratt ${ }^{1}$, J. Weese ${ }^{2}$, and D.J. Hawkes ${ }^{1}$ \\ ${ }^{1}$ Centre for Medical Image Computing, Department of Medical Physics \& \\ Bioengineering, University College London, UK \\ I.Waechter@cs.ucl.ac.uk \\ ${ }^{2}$ Philips Research Aachen, Germany
}

\begin{abstract}
For assessment of cerebrovascular diseases, it is beneficial to obtain three-dimensional (3D) information on vessel morphology and hemodynamics. Rotational angiography is routinely used to determine the 3D geometry and we propose a method to exploit the same acquisition to determine the blood flow waveform and the mean volumetric flow rate. The method uses a model of contrast agent dispersion to determine the flow parameters from the spatial and temporal development of the contrast agent concentration, represented by a flow map. Furthermore, it also overcomes artifacts due to the rotation of the c-arm using a newly introduced reliability map. The method was validated on images from a computer simulation and from a phantom experiment. With a mean error of $11.0 \%$ for the mean volumetric flow rate and $15.3 \%$ for the blood flow waveform from the phantom experiments, we conclude that the method has the potential to give quantitative estimates of blood flow parameters during cerebrovascular interventions.
\end{abstract}

\section{Introduction}

For diagnosis, treatment planning and outcome control of cerebrovascular diseases, such as atherosclerosis, aneurysms, or arteriovenous malformations (AVM), it is beneficial to obtain three-dimensional (3D) information on vessel morphology and hemodynamics. Rotational angiography (RA) allows 3D information on vessel morphology to be obtained during an intervention and is used routinely at the beginning of aneurysm and AVM interventions. As it is possible to see how the contrast agent moves with the blood, the sequence contains hemodynamic information as well. This information, however, is currently not exploited in clinical systems. The goal of this work was to quantify the blood flow waveform and the mean volumetric flow rate from rotational angiography data.

Several different approaches for the determination of blood flow information from planar (not rotating) angiography have been reported previously. All existing approaches are based on analyzing changes in the x-ray image intensity due to changes in the contrast agent concentration. Shpilfoygel et al. 1] give a comprehensive review of established techniques, which include:

- techniques based on bolus tracking using time-intensity curves (TICs) at different sites along a vessel 
- techniques based on distance-intensity curves (DICs) at different points in time;

- and techniques based on optical flow.

Methods based on bolus tracking are known to give unreliable results in the case of pulsatile flow [12, and it is not possible to extract a time dependent waveform. Methods based on DICs and on optical flow enable a time-dependent waveform to be extracted. They, however, are sensitive to noise and the flow estimate depends on the distance to the injection site and the time from the start of the injection due to the effects of convective dispersion [2 3]. We propose a model-based flow estimation to overcome these problems by explicitly modeling convective dispersion.

To measure absolute flow parameters, it is necessary to know the 3D geometry of the vessel system, which cannot be obtained from a planar scan. As the 3D geometry can be obtained from RA, it is an advantage to extract the flow information from the same sequence. In this case only one contrast agent injection is required. Recently, there have been two approaches to achieve this: Chen at al. use a bolus tracking method [4, whereas Appaji and Noble use an optical flow method to reconstruct flow from RA [5]. However, both approaches do not address artifacts introduced by the rotation of the c-arm. In this paper, we propose a model-based flow estimation for RA, which addresses this problem.

\section{Method}

Our method first determines the so-called flow map and the newly introduced reliability map from the RA image sequence. The flow map is represented as an image, where the intensity is the concentration of contrast agent, the horizontal dimension is time and the vertical dimension is length along the vessel. Then a simulated flow map is fitted to the extracted flow map. The simulated flow map is generated using a model based on the physics of blood flow and contrast agent

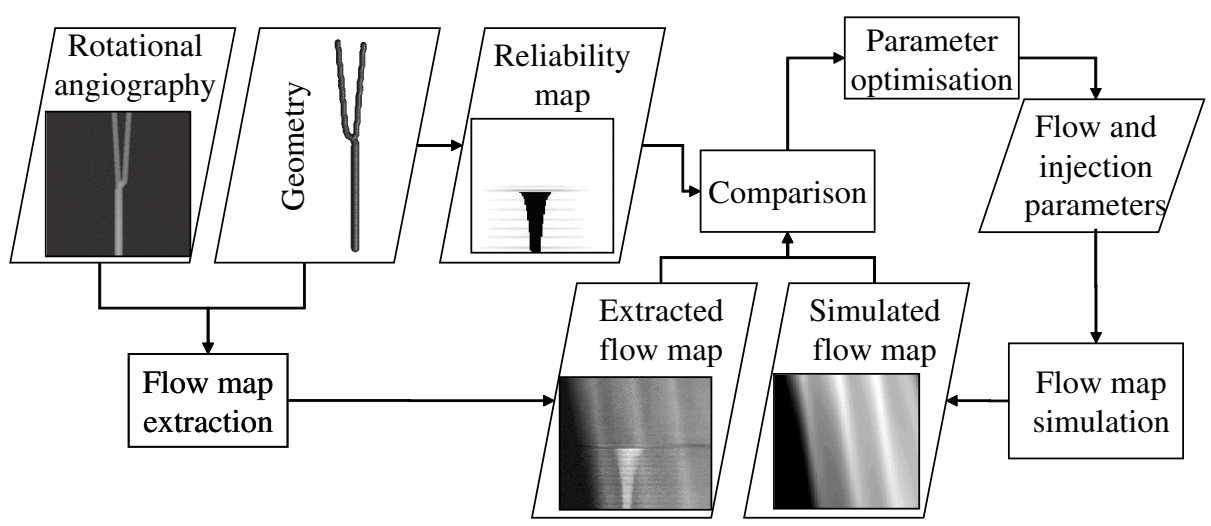

Fig. 1. Overview of the flow map fitting 
transport. During the fitting, the optimal parameters for blood flow, including the flow waveform, and for the contrast agent injection are determined. An overview is given in Fig. 1 and all components are described in detail below.

\subsection{Extraction of Flow Maps and Reliability Maps}

At first the RA images are preprocessed to give quantitative information about the amount of iodine on the x-ray path. This can be done by digital subtraction of a mask scan and iodine calibration [6]. The RA sequence consists of $M$ preprocessed images $I(x, y, t)$, acquired at the time steps $t \in[1,2, \ldots, T]$ at different geometrical configurations $G(t)$ of the rotating c-arm.

Then, the $3 \mathrm{D}$ vessel centerline and radii are determined from the $\mathrm{RA}$ sequence as described in [7. This method was tailored for RA sequences which show inflow and outflow of contrast agent. If $l \in[1,2, \ldots, L]$ represents the length along the vessel, the centerline is given by a set of $3 \mathrm{D}$ points $(x, y, z)=P(l)$ and for each point the corresponding radius is given by $R(l)$. The projection function $\Pi(P(l), G(t))$ relates the positional co-ordinates of the $3 \mathrm{D}$ point $P(l)$ to the coordinates of the corresponding pixel within the x-ray image acquired at time $t$. A geometric calibration is used beforehand to determine the geometry parameters $G(t)$.

The uncorrected flow map $\mathbf{F}_{U}(l, t)$ is determined by projecting each $3 \mathrm{D}$ point of the centerline onto each image of the RA sequence and is given by

$$
\mathbf{F}_{U}(l, t)=I(\Pi(P(l), G(t)), t) .
$$

Due to the rotation of the x-ray system, the flow map contains artifacts produced by two effects: Foreshortening and vessel overlap. In a projection image, vessels appear foreshortened if the direction of the x-ray is not perpendicular to the vessel. Vessels appear foreshortened frequently during a RA sequence. Vessel overlap occurs when more than one vessel segment lies on the x-ray beam path. The foreshortening error can be partially corrected: For every point $P(l)$ and every configuration of the c-arm $G(t)$, the length of the intersection of x-ray beam and current vessel is given by $\mathbf{L}(l, t)$ determined from the centerline and radii. This length is used for the correction of the flow map (referred to as extracted flow map) as given by

$$
\mathbf{F}_{E}(l, t)=\frac{\mathbf{F}_{U}(l, t)}{\mathbf{L}(l, t)} .
$$

As the amount of contrast agent is divided by $\mathbf{L}(l, t), \mathbf{F}_{E}(l, t)$ represents the distribution and time course of the concentration of iodine. However, the information of foreshortened vessels is still less reliable and errors due to overlapping vessels cannot be corrected. The reliability $\operatorname{map} \mathbf{R}_{F}(l, t)$ is used to overcome this problem. The reliability of each entry of the flow map is given by

$$
\mathbf{R}_{F}(l, t)= \begin{cases}0 & , \text { if vessel overlap is detected } \\ \cos \theta, & \text { otherwise, }\end{cases}
$$


where vessel overlap is defined as the case when more than one vessel segments intersects with the x-ray beam and $\theta$ is the angle between the x-ray beam and the vessel centerline, which gives the degree of foreshortening.

\subsection{Model-Based Flow Map Simulation}

The model is used to predict a flow map, given the parameters of the blood flow and the injection. The blood flow is modeled by

$$
Q_{b}(t)=\bar{Q}_{b} \cdot w(t),
$$

where $\bar{Q}_{b}$ is the mean volumetric flow rate and $w(t)$ is the waveform

$$
w(t)=\frac{\delta+\cos \left(2 \pi \cdot f_{\alpha, \beta, \gamma}(t / T)\right)}{\int_{0}^{1} \delta+\cos \left(2 \pi \cdot f_{\alpha, \beta, \gamma}(x)\right) d x},
$$

where $\delta$ gives the baseline flow and $f_{\alpha, \beta, \gamma}(t):[0,1] \rightarrow[0,1]$, with $f(\alpha)=$ $a, f(\beta)=b, f(\gamma)=c,(a, b, c$ fixed), is a piecewise linear, monotonic function. $\alpha, \beta, \gamma$ specify the shape of the waveform. The injection flow $Q_{i}(t)$ is assumed to be a rectangular function, defined by the maximum flow $\widetilde{Q}_{i}$, the start time $T_{s}$ and duration $T_{d}$ of the injection. The energy loss in the catheter is modeled by a lag element with characteristic time $T_{l}$. Mixing is assumed to be uniform. The concentration of iodine at the injection site is the given by

$$
\mathbf{C}(r, 0, t)=c \cdot \frac{m \cdot Q_{i}(t)}{Q_{b}(t)+m \cdot Q_{i}(t)},
$$

where $m \leq 1$ is a mixing factor and $c$ is the concentration of iodine in the contrast agent. The flow is assumed to be laminar and axially symmetric. The vessel is divided in $N$ laminae with average radius $r_{n}, n \in[1,2, \ldots, N]$. The velocity distribution in each laminae depends on the flow profile $p\left(r_{n}\right)$. A profile, given by

$$
p\left(r_{n}\right)=1-\left(r_{n} / R\right)^{k}, 2 \leq k<\infty
$$

can approximate a profile between plug flow $(k \rightarrow \infty)$ and parabolic flow $(k=2)$. The velocity in laminae $n$ is then given by

$$
v\left(r_{n}, t\right)=v_{0}(t) \cdot p\left(r_{n}\right)=\frac{Q_{b}(t)+m \cdot Q_{i}(t)}{2 \pi \sum_{n=1}^{N} p\left(r_{n}\right) r_{n}} \cdot p\left(r_{n}\right) .
$$

The transport of a soluble substance in a moving medium is determined by convection and diffusion [3]. Using the velocity of the laminae, the convection of iodine is then determined in $2 \mathrm{D}$ representation of the vessel tree, parameterized in terms of length $l$ along and distance $r$ from the vessel centerline [2]. Diffusion is modeled by applying a Gaussian low pass filter. The result is the time-dependent development of the concentration of iodine $\mathbf{C}(r, l, t)$. Finally, the simulated flow map is given by

$$
\mathbf{F}_{S}(l, t)=\frac{1}{R} \sum_{n=1}^{N} \mathbf{C}\left(r_{n}, l, t\right) .
$$




\subsection{Flow Map Fitting}

For the flow map fitting, the simulated flow maps $\mathbf{F}_{S}$ are fitted to the extracted flow map $\mathbf{F}_{E}$. The error between them is determined by

$$
E\left(\mathbf{F}_{S}\right)=\sum_{l=1}^{L} \sum_{t=1}^{T}\left[\left(\mathbf{F}_{S}(l, t)-\mathbf{F}_{E}(l, t)\right)^{2}+\left(\nabla\left(\mathbf{F}_{S}(l, t)\right)-\nabla\left(\mathbf{F}_{E}(l, t)\right)\right)^{2}\right] \cdot \mathbf{R}_{F}(l, t),
$$

where $\nabla$ is the temporal gradient operator and the reliability map gives a weighting. A Gauss-Newton least-squares optimization is used to determine the injection and flow parameters, in particular, the waveform parameters $\alpha, \beta, \gamma$,and $\delta$ and the mean volumetric flow rate $\bar{Q}_{b}$ by minimizing $E\left(\mathbf{F}_{S}\right)$.

\subsection{Validation}

The proposed method for flow extraction was validated by computer simulations and a phantom experiment. Three setups were used to determine the extracted flow map as follows:

1. Simulated flow maps were corrupted by different levels of noise (Peak signalto-noise ratio: $18,22,28$ ) and used as extracted flow maps. The flow and injection parameters were varied according to Table 1 . Three different waveforms (Fig. 2) were used. Overall 272 different flow maps were tested.

2. Flow maps were extracted from computer simulated RA. The contrast agent propagation was based on the model described above. The resulting concentrations $\mathbf{C}(r, l, t)$ were mapped to a voxel presentation of the vessel tree and converted to $\mathrm{x}$-ray attenuation coefficients. The $\mathrm{x}$-ray images were determined using a proprietary, analytical x-ray simulation software. Two example geometries were used: The first geometry was a curved tube with uniform radius, which produced a foreshortening artifact (see Fig. 3), and the second was a bifurcation, which produced an overlapping artifact(see Fig. (1). Flow and injection parameters were chosen as above.

3. Flow maps were extracted from the images from an experimental setup. The experimental setup consisted of a pulsatile flow circuit, a clinical contrast agent injector (MarkVProVis, Medrad) and a rotational x-ray system (Allura Xper with an FD20 detector, Philips Medical Systems). Seventeen image sequences were acquired following a contrast injection (Ultravist-370, Schering) into a straight tube. The tube was placed in an elliptical, water filled cylinder to generate realistic noise, beam hardening, and scatter. The ground truth flow was acquired by an electromagnetic flow meter (EMF). The flow parameters were estimated from a full length flow map $(10 \mathrm{~cm})$ and from a short flow map $(1 \mathrm{~cm})$.

The flow waveform was determined from each flow map using the flow map fitting method described in Sec. 2.3 and compared with the ground truth waveform. The waveform was initialized to a cosine-function and the mean volumetric flow rate was either initialized at half of the desired value or randomly chosen. 
Table 1. Parameters of simulation

\begin{tabular}{|c|c|c|c|}
\hline Parameters & Symbol & Unit & Values \\
\hline Mean volumetric flow rat & $Q_{b}$ & {$[\mathrm{ml} / \mathrm{min}]$} & {$[100-300]$} \\
\hline Flow profile & $k$ & {$[1$} & {$[2,5,10]$} \\
\hline Radius & $R$ & {$[\mathrm{~mm}]$} & {$[2]$} \\
\hline Max injection flow rate & $\widetilde{Q}_{i}$ & {$[\mathrm{ml} / \mathrm{min}]$} & {$[50-300]$} \\
\hline Injection lag & $T_{l}$ & {$[\mathrm{~s}]$} & {$[0.2-0.5]$} \\
\hline Iodine concentration & $c$ & {$[\mathrm{mg} / \mathrm{ml}]$} & {$[370]$} \\
\hline Start injection & $T_{s}$ & {$[\mathrm{~s}]$} & {$[0,0.5,1]$} \\
\hline Injection duration & $T_{d}$ & {$[\mathrm{~s}]$} & {$[3,4]$} \\
\hline Mixing factor & $m$ & [] & {$[1]$} \\
\hline Acquisition duration & $T$ & {$[\mathrm{~s}]$} & {$[4]$} \\
\hline Total number frames & $M$ & [] & {$[120]$} \\
\hline Number Laminae & $N$ & [] & {$[10]$} \\
\hline
\end{tabular}
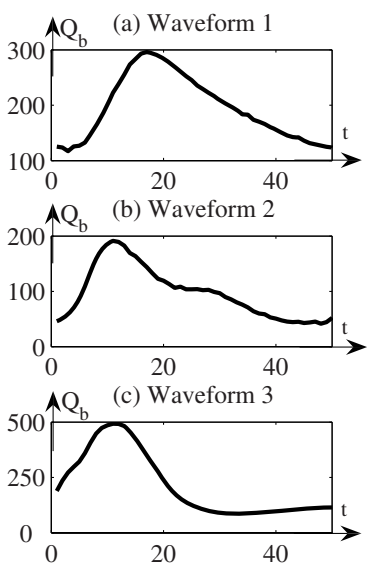

Fig. 2. Waveforms

The percentage error in the mean volumetric flow rate (mean flow) and waveform were calculated.

\section{Results}

The results of the three experiments were as follows:

1. For the first setup, the mean error of the mean flow and of the waveform were $0.7 \%$ and $7.3 \%$, respectively. The accuracy of the waveform mainly depended on the shape of the wave form (Waveform 1: 4.5\%, Waveform 2: $7.6 \%$, Waveform 3: 9.8\%; other flow and injection parameters had only

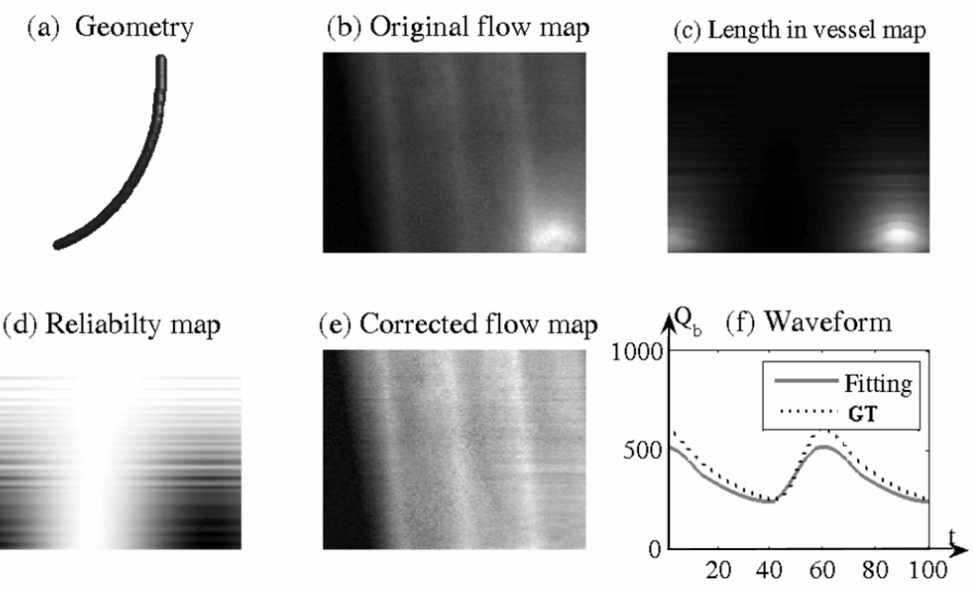

Fig. 3. Results from the computer simulation using the foreshortening phantom 
(a) Extracted flow map

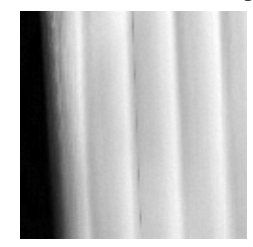

(b) Simulated flow map

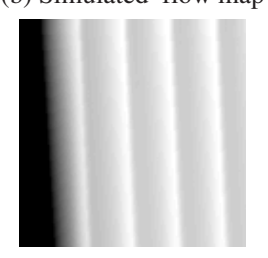

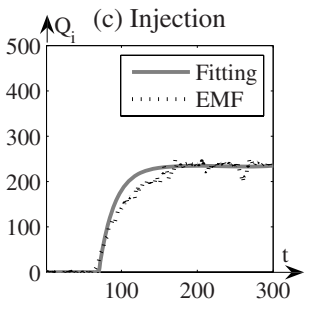

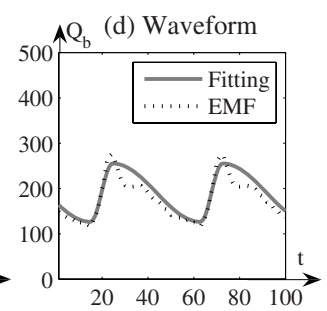

Fig. 4. Results from the experimental setup

minor influences. The influence of noise on the accuracy is relatively small (Waveform 1: No noise: 4.7\%, low noise level: 4.5\%, medium noise level: $4.6 \%$, high noise level: $5.1 \%$ ).

2. For the bifurcated geometry, the mean error of the mean flow and waveform were $11.8 \%$ and $16.4 \%$, respectively. For the curved geometry, the mean error of the mean flow and waveform were $9.8 \%$ and $14.6 \%$, respectively. Fig. 3 shows the uncorrected flow map, reliability map and corrected flow map for the curved geometry. Fig. 1]includes some results for the bifurcated geometry.

3. For the full length flow map from the experimental setup, the mean error of the mean flow and the waveform were $11.0 \%$ and $15.3 \%$, respectively. For the short flow map, the error increased only to $13.0 \%$ and $17.3 \%$. An example of a full length extracted flow map, the according fitted flowmap and estimated waveform together with the ground truth waveform are given in Fig. 4.

The optimization converged to an accurate solution, even when the start parameters were far away from the desired result parameters. It took 10 to 20 iterations for the convergence. The flow map fitting for the extraction of one waveform took about $5 \mathrm{~min}$ on a $3 \mathrm{GHz}$ Xeon processor.

\section{Discussion}

In this paper, we have described a method for estimating a blood flow waveform and the mean volumetric flow rate from RA. Any method estimating blood flow from RA must be more robust than for planar angiography, because of image artifacts introduced by the rotation. To achieve this, we have introduced a novel approach, that utilizes a model-based flow estimation and the reliability map.

The reliability map is used to decrease the influence of artifacts on the flow estimation and to use as many segments of the vessel tree as possible. Existing methods use either only DICs (columns of the flow map) or TICs (rows of the flow map). Methods based on DICs in particular require a certain length of vessel to be unoccluded or foreshortened. In the case of rotational data and a non-trivial vessel geometry, it is not possible to determine sufficiently long, artifact-free DICs. However, even in complex geometries, enough segments should be not foreshortened and not overlapped at least in some parts of the RA sequence. The reliability map makes it possible to use this information. 
The flow map fitting uses the flow map as a whole rather than single TICs or DICs. Therefore, it is more robust against noise and uncorrected artifacts compared with existing methods. The first experiment showed that even high levels of noise introduced only small errors into the waveform estimation.

A further advantage is that the proposed method incorporates a model of the underlying physics. Methods based on optical flow and DICs on the other hand have the disadvantage that the flow estimation changes with the distance from the injection site and time from the start of the injection. Our method models the convective dispersion and can therefore account for these different situations.

The last experiment showed that the flow model is able to explain real transport of contrast agent in a flowing medium. With a mean error of $11.0 \%$ for the mean volumetric flow rate and $15.3 \%$ for the blood flow waveform from the phantom experiments, we conclude that the method has the potential to give sufficiently accurate, quantitative estimates of blood flow parameters.

Such quantitative data can supplement the subjective information on flow currently obtained by visual interpretation during cerebrovascular interventions. For application of the method to clinical data, the frame rate of the RA sequence should be at least $30 \mathrm{fps}$. The distance between the injection site and the first observation should be known. Furthermore, a iodine calibration is beneficial. For the future, we plan experiments with more complex geometries and a comparison to a computational fluid dynamics simulations.

We thank the X-ray Pre-Development department at Philips Medical Systems Best for making the experiments possible.

\section{References}

1. Shpilfoygel, S.D., Close, R.A., Valentino, D.J., Duckwiler, G.R.: X-ray videodensitometric methods for blood flow and velocity measurement: A critical review of literature. Med. Phys. 27(9), 2008-2023 (2000)

2. Rhode, K.S., Lambrou, T., Hawkes, D.J., Seifalian, A.M.: Novel approaches to the measurement of arterial blood flow from dynamic digital X-ray images. IEEE Trans. Med. Imaging 24(4), 500-513 (2005)

3. Taylor, G.: Dispersion of soluble matter in solvent flowing slowly through a tube. In: Proceedings of the Royal Society of London. Series A-Mathematical an physical science, vol. 219(1137), pp. 186-203 (1953)

4. Chen, Z., Ning, R., Conover, D., Lu, X.: Blood flow measurement by cone-beam CT bolus imaging. In: Medical Imaging, Proceedings of the SPIE (2006)

5. Appaji, A., Noble, A.J.: Estimating cerebral blood flow from a rotational angiographic system. In: Biomedical Imaging: Macro to Nano, pp. 161-164 (April 6-9, 2006)

6. Molloi, S., Bednarz, G., Tang, J., Zhou, Y., Mathur, T.: Absolute volumetric coronary blood flow measurement with digital subtraction angiography. Int. J. Card Imaging 14(3), 137-145 (1998)

7. Waechter, I., Bredno, J., Weese, J., Hawkes, D.: Using flow information to support 3D vessel reconstruction from rotational angiography. Medical Physics 33, 1983 (2006) 